\title{
El aporte de la historia y teoría del diseño al taller de diseño industrial ${ }^{1}$
}

\author{
The contribution of Design History and Theory to the \\ Industrial Design workshop
}

Cómo citar:

Ortiz, J., Supisiche M. \& Savogin, G. (2019). El aporte de la historia y teoría del diseño al taller de diseño industrial. Designia, 6 (2), 55-83.

\section{Palabras clave:}

Formación, enseñanza en equipo, discusión.

*Arquitecto de la Universidad Católica de Santa Fe y Magíster en Arquitectura Mención en Teorías de la Arquitectura Contemporánea en la Universidad Nacional del Litoral. Docente en el área de "Historia de la Arquitectura y el Urbanismo I y II" en la carrera de Arquitectura,

e "Historia del Diseño Industrial y el Arte" / "Historia de la Industria Argentina, Sociedad y Cultura" en la Licenciatura en Diseño Industrial

en la UCSF. Publicación de libros y artículos en revistas científicas: "AREA" de UBA; "RDA" de UCSF.

E-mail: juanceor@outlook.com ORCID: http://orcid.org/0000-0002-8427-0774

**Arquitecta de la Universidad Católica de Santa Fe y Magíster en Conservación del Patrimonio UCSF. Docente Emérita UCSF 2013-2019. Investigadora Categoría A, UCSF. Evaluadora de Proyectos de Investigación. Miembro de Jurado de concursos docentes en sus distintas categorías UCSF. Publicación de libros y artículos en revistas científicas: "AREA" de UBA; "RDA" de UCSF. E-mail: mc@supisiche.com.ar ORCID: https://orcid.org/0000-0002-5716-8627

***Diseñador Industrial por la Universidad Nacional de Córdoba con experiencia profesional en gestión de diseño y desarrollo de productos

en la industria automotriz, médica, de electrodomésticos, metal mecánica, muebles, de herramental y matricería. Creador y Director de la Carrera Licenciatura en Diseño Industrial de la UCSF. Profesor de Diseño Industrial I. E-mail: gsavogin@ucsf.edu.ar ORCID: https://orcid.org/0000-0003-0953-4829

\section{Key words:}

Education, team teaching, argumentation.

Recibido: 08/03/2019

Aceptado: 01/05/2019 
En el presente artículo de investigación se plantean, desde una mirada teóricopráctica, las dificultades que se vislumbran en la enseñanza-aprendizaje de la historia y teoría del diseño industrial, referidas al proceso proyectual realizado en los talleres de diseño industrial de la universidad a la que pertenece este equipo de investigación, la Universidad Católica de Santa Fe.

Se tiene la convicción de que en la formación del diseñador industrial el conocimiento de disciplinas como las mencionadas ut supra son vitales para el adecuado ejercicio profesional, ya que les proporcionan competencias que les permiten percibir, concebir y delinear proyectos de objetos más acabados, como simulacro de la actuación profesional futura.

El propósito esencial de este trabajo de investigación es propiciar la comprensión e internalización de las variables del proyecto de Diseño Industrial, entendiendo a la historia y a la teoría del diseño como un conjunto de conocimientos considerados como insumos ${ }^{2}$ para el desarrollo, argumentación y realización del proyecto de diseño.

\section{Abstract:}

This article raises, from a theoretical-practical perspective, the difficulties that are glimpsed in the teaching-learning of history and theory of Industrial Design, referred to the design process accomplished in the Industrial Design Workshops of the university to which this research team belongs.

The conviction is that in the formation of the Industrial Designer the knowledge of disciplines such as those mentioned above are vital for the adequate professional practice since they provide with skills that allow to perceive, conceive and delineate projects of more finished objects, as a simulation of the future professional performance.

The essential purpose of this research is to promote understanding and internalization of the variables of the Industrial Design project, understan-

${ }^{2}$ Insumo en el sentido operativo, que mediante la reflexión y la crítica de los fenómenos de la historia y la teoría aporta criterios proyectuales. ding history and theory as a set of knowledge considered as inputs for the development, argumentation and realization of the project design.

e-book. It is thus intended reducing the digital gap, fostering this type of content, making it more accessible and responding to the needs identified when working with the School's young population. 


\section{INTRODUCCIÓN}

La experiencia docente de este equipo de investigación ha detectado una parcial vinculación entre los talleres de diseño y los contenidos de historia y teoría del diseño en la carrera de Licenciatura en Diseño Industrial a la que pertenecen, y durante la indagación bibliográfica se ha observado que ese problema es común tanto en instituciones de Argentina como en otros países de Latinoamérica.

Tanto en los docentes como en los estudiantes se aprecian criterios diversos en la consideración del deber ser del diseño industrial, los cuales se considera importante evidenciar en esta investigación.

Ochoa y Agusto (2014) sostienen que, en la formación de diseñadores industriales, las ciencias sociales, y especialmente la historia, se enfrentan a una realidad singular. El estudiante de primer año presenta una definida voluntad de dibujar, modelar y crear, pero en todos los casos la resistencia a la lectura es expresa.

Esta resistencia es un desafío por afrontar de modo particular, a través de estrategias que posibiliten al estudiante hacerse obra de sí mismo, generando proposiciones concretas a fin de educar sin 'fabricar', así como también descubriendo caminos alternativos que le permitan al estudiante moverse de su posición de mero espectador al espacio de protagonista, logrando de este modo que 'haga' de la historia una herramienta más para materializar sus ideas.

Otra mirada importante es la de Zamora Sarmiento (2015), quien refiere a un cambio en el ejercicio del diseño. El diseñador deja su rol como artista creativo y asume los retos de entender al usuario, contexto y demás variables que intervienen en los proyectos de diseño. 
¿Cómo se está adaptando la enseñanza a esto? Se resalta la importancia del trabajo articulado del diseñador en el proceso de comprensión de problemáticas, convirtiéndolo en interlocutor que escucha, analiza, interpreta y propone con expertos de otras áreas, a partir del entendimiento de problemas socialmente determinantes y con análisis complejos que llevan a múltiples soluciones relevantes, coherentes y pertinentes respecto a los usuarios y contextos.

La historia y la teoría del diseño industrial proporcionan algo fundamental en todo proceso creativo: el conocimiento de los diferentes contextos en los cuales se han realizado las intervenciones culturales en el mundo natural. Sin esta mirada abarcadora y en profundidad, las nuevas propuestas carecen de una sólida base de sustentación, ya que nada se genera a partir de la nada.

Como se afirma en el documento de UNESCO (1980) referido al juego de los niños, la historia permite leer el pasado y el presente de las sociedades y de los pueblos, por lo cual es ineludible atender lo que aquella y sus correspondientes teorías dicen, para anclar firmemente la producción.

Por tanto, se considera necesario insistir en la idea de que el estudio y reflexión crítica de la historia y la teoría del diseño industrial, en torno a los fenómenos que hayan implicado cambios artificiales del hábitat, aportarán la experiencia ineludible para la materialización de futuras acciones de diseño, ya que todo este proceso, compuesto por una sucesión de disposiciones conectadas entre sí, necesita de la materia prima de la historia y la teoría para la preparación de juicios de diseño que asistan a la toma de decisiones.

Se debe comprender que la historia y la teoría del diseño industrial son disciplinas recientes, que se determinan como espacio concreto competente recién en el siglo XX. No obstante, los juicios podrían asociarse, por su carácter de aproximación, con lo sostenido por Kruft (1990), cuando se refería a la historia de la teoría de la arquitectura como "la suma de aquello que ha sido formulado expresamente como teoría de la arquitectura" (p. 13).

Se aborda esta investigación con la convicción de que la producción de conocimientos dilata las fronteras de una disciplina. La indagación apoyada en los caminos de la ciencia busca, indaga, interpreta, idea, crea, modifica, transforma el conocimiento con método, y desde ahí explora la realidad, resuelve o anticipa situaciones. En sentido exacto es sistémica, objetiva, verificable, experimental y formal. Al vincularse al proceso educativo le comunica a este rigor, reflexión, método y autonomía. 
Surgen aquí las siguientes preguntas: ¿La investigación en diseño existe? ¿Cuál es? ¿Cuál es su objeto? ¿Cuál es su método? ¿El diseño puede producir nuevo conocimiento?

Sostiene Guigosa Fragoso (2006), citando a Joan Costa, que "muchos confunden todavía los métodos con las técnicas, cuando en realidad los métodos sirven a la estrategia para pensar y planear, y las técnicas sirven para 'hacer'" (pp. 76-77). Los conocimientos teóricos enriquecen y fundamentan el proyecto de diseño, proceso distintivo y diferenciador de la disciplina. Se parte de la premisa de que el diseño sigue un proceso iterativo, conformado básicamente por dos aspectos, uno racional (proceso racional) y otro creativo (proceso creativo). En este sentido y como actividad fáctica, necesita de la investigación para el desarrollo de metodologías de diseño en búsqueda de la sistematización de la práctica y una mayor objetividad.

El estudio, registro y reflexión crítica del desarrollo histórico de los hechos, fenómenos y objetos de naturaleza proyectual en las áreas del diseño industrial que impliquen cualquier cambio, tendrá como objetivo aportar a la práctica del diseño toda la experiencia primaria necesaria para la concreción de acciones futuras mediante el proceso de razonamiento pertinente.

Todo proceso de diseño está constituido por una serie de decisiones concatenadas, y en este sentido el conocimiento de la historia del diseño es útil como materia prima que permita la elaboración de criterios de diseño que ayuden a la toma de decisiones.

La teoría del diseño, en el ámbito del proyecto, aporta un conocimiento profundo de las ideas puestas en juego en la creación de la forma, aunque se puede afirmar que todo producto de diseño se basa en principios teóricos que no han de estar necesariamente verbalizados. Hanno Kruft (1990) plantea que los sistemas que se presentan como teorías integran categorías estéticas, sociales y prácticas, abarcando 
así un espectro amplio de variables a considerar, las cuales, análogamente, se verifican en el espacio del diseño industrial.

La teoría del diseño resulta imprescindible como fundamento para el proyectista en ejercicio si este quiere tener claridad con respecto a los principios con los que trabaja. Para dilucidar la propia situación es necesario, o al menos útil, saber cómo otros han abordado problemas análogos.

A través de la historia del diseño industrial y la historia de la teoría del diseño se observa cómo el hombre responde ante las exigencias culturales de cada época, aportando una visión esclarecedora de los fundamentos que llevan al diseñador a tomar determinadas decisiones proyectuales, evaluando así aciertos y yerros. El fin fundamental de este trabajo es favorecer el conocimiento de estas variables del proyecto de diseño, delimitadas en su momento cultural, que pueden esclarecer análogamente y de manera ejemplar los complejos procesos de diseño contemporáneos.

La importancia del conocimiento de la historia y de los ejemplos de diseño de objetos como referencia radica en que esta actúa como instrumento didáctico ejemplar. El diseño se aprende desde la propia experiencia trazada por profesionales destacados, de sus valores y sus técnicas. Siguiendo a Ferrer Forés (2009), la profundización en el proyecto de referencia establece vínculos más estrechos y profundos con el pasado, haciéndolos funcionales a los problemas de diseño del presente.

De esta manera se propone un enfoque integrador interdisciplinario, considerando la historia no como un banco de información de tipo empírico-descriptivo, descontextualizado y des-teorizado, sino como un trasfondo de experiencias valiosas que puede ayudar a comprender y a interpretar, $\mathrm{y}$, por ende, enfocar mejor los problemas de diseño contemporáneos.

Por tanto, este equipo de investigación presenta los siguientes objetivos e hipótesis:

\section{Objetivo general}

- Resolver la falta de transferencia de los insumos proporcionados por la historia del diseño industrial y la teoría del diseño al proceso proyectual en taller.

\section{Objetivos particulares}

- Analizar y valorar la incidencia de los conceptos trabajados como historia del diseño industrial en la producción proyectual de los estudiantes del taller de diseño correspondiente. 
- Analizar y valorar la incidencia de los diferentes momentos históricos y sus correspondientes teorías del diseño en la producción de objetos durante el siglo XX, en función de las diversas corrientes estéticas.

- Aportar estrategias de abordaje del proceso de diseño, tomando las referencias históricas y teóricas didácticamente.

\section{Objetivo de acción}

- Diseñar con los docentes de los talleres de diseño estrategias de abordaje del proceso proyectual, tomando las referencias históricas y teóricas como insumos didácticos (insumo en el sentido operativo del término, que mediante la reflexión y la crítica de los fenómenos específicos de la historia aporta criterios proyectuales, como se afirmó ut supra).

\section{Hipótesis}

- La historia y la teoría del diseño industrial como referencia pueden mediar como instrumentos didácticos operativos en el marco del proceso de diseño, ya que este se aprende desde la propia experiencia y la tradición, desde sus valores, sus técnicas y su dimensión interdisciplinar.

\section{Marco teórico}

Sostiene Maldonado (1997) que "el Diseño Industrial no es un fenómeno de naturaleza aerolítica, algo insólito e inexplicable en la historia, sino al contrario, es un fenómeno que deriva de recorridos de la cultura del pasado, y que apunta a objetivos claros y definidos" (p. 63). En otro lugar, el mismo autor afirma que:

... el Diseño es una actividad proyectual dirigida a determinar las propiedades formales de los objetos producidos industrialmente. Por propiedades formales no se deben deducir solamente las características exteriores, sino, sobre todo, las relaciones funcionales y estructurales que hacen de un objeto una unidad coherente, tanto desde el punto de vista del producto como del usuario. Propiedades que resultan de la integración de factores múltiples: funcionales, culturales, tecnológicos y económicos. (1961, en Bonsiepe, 1999, p.35) 
Quizás la errónea interpretación de esta aproximación conceptual, en su postulado inicial, sea la razón por la cual la enseñanza del diseño ha estado orientada generalmente hacia la formación de habilidades y capacidades, como se enseña más un oficio, antes que hacia la construcción de un corpus teórico específico y funcional a la tarea disciplinar.

Así, la superficialidad aparente en la que se mueve la resolución proyectual como tarea mecánica con escaso sustento teórico resulta una conclusión frecuente en el ámbito académico formativo, donde el método de enseñanza supone una instancia de simulación en la que se aprende haciendo, con lo cual se obtienen respuestas poco reflexivas que restringen las posibilidades de búsqueda, las posturas críticas y la autonomía disciplinar. La investigación en diseño, en realidad, adolece de un estatus mejor definido y sigue siendo motivo de discusión.

Si se parte de la idea de que el proyecto de diseño es también a su modo una investigación, entonces la historia del diseño industrial y la teoría se convierten en algo capaz de incorporarse operativamente a esta investigación, con la ventaja de permitir la observación de los hechos pasados con una mayor distancia que actúa como filtro y los aclara.

De este modo, proyectar con los productos industriales ejemplares del pasado próximo, dada la juventud de la disciplina, ejerce una intensa dimensión operativa en un marco de integración entre las áreas de la historia y las de taller. El diseño se aprende desde la propia disciplina, de sus valores y sus técnicas, como también de la interdisciplinariedad. El ahondamiento en el proyecto de referencia establece vínculos más estrechos y profundos con el pasado.

En el Taller de diseño se aspira a un aprendizaje gradual y paulatino, instruyendo para valorar y reconocer los mecanismos operativos de que se vale el diseñador; en definitiva, se trata de descubrir y cultivar la mirada y el análisis siguiendo al producto industrial como instrumento constructivo. La enseñanza de la historia y la teoría del diseño pueden apoyar esta tarea.

A su vez, la teoría es una actividad generadora de argumentos de proyecto, en tanto arsenal de dispositivos intelectuales y de ejercitación de posibilidades de acción en cualquier área del diseño. No se puede diseñar sin sentido, como no se puede proyectar sin fundamentos.

La teoría y los argumentos constituyen entonces el modo conductor del diseño hacia un aspecto consciente y propositivo del proyecto. 
No se cree que la teoría sirva en términos de un conocimiento intelectual como mero instrumento de erudición, sino que debe estar enfocada hacia la principal actividad del proyectista: diseñar, resolver problemas, tanto funcionales como estéticos y/o simbólicos, en los productos que propone.

Tampoco se debe confundir la teoría con la crítica o con la historia del diseño. La teoría es una actividad de proposición a partir de la reflexión, de la contemplación, pero no de construcción de conceptos abstractos de uso clasificatorio. La teoría es acción erudita, está adentro del proyecto, adentro de la disciplina activa.

En síntesis, el alumno que inicia los estudios de diseño industrial necesita adquirir hábitos intelectuales, visuales, perceptivos y gráficos, y desarrollarlos gradualmente en el transcurso de su trayectoria académica. Para esto es necesario el conocimiento, desde la historia, de los ejemplos que desde un pasado próximo explicitan tanto valores como técnicas de proyección y construcción de objetos sujetos a producción industrial. Se concibe a los objetos industrializados como indicadores sociales, no solo por su efecto económico, sino porque expresan una forma de recrear la realidad de un tiempo específico: sirven para hacer el mundo más inteligible, para mejorar la vida de las personas y para hacer más comprensible el entorno, entre otras cosas. Se coincide con García Trompa (2006) cuando sostiene que los:

\footnotetext{
... objetos de diseño se conforman por pensamientos y no por formas que estructuran identidad. La identidad de un grupo cultural no está mediada únicamente por toda manifestación formal existente; estas lo sustentan, pero no son estructura sólida en la cual pueda apoyarse un diseño. Más allá de un gesto característico, de una técnica, de un material característico; nos diferencia de todos, un modo de pensar característico. (p. 106)
}

Así la historia y la teoría del diseño industrial refuerzan y ayudan a desarrollar la cultura del proyecto, pensando desde el pasado las múltiples implicaciones que cada 'objeto' diseñado o por diseñar representa, según lo sostenido por García Trompa (2006). La investigación, la reflexión y la crítica resultan herramientas fundamentales para contribuir de modo eficiente a la construcción del problema de diseño en taller. 
El entonces ICSID (International Council of Societies of Industrial Design, o Consejo Internacional de Sociedades de Diseño Industrial), actualmente WDO (World Design Organization, u Organización Mundial del Diseño), elaboró hacia 2005 una nueva definición del diseño industrial (Reinders, Diehl y Brezet, 2013), donde se lo considera como una actividad creativa cuyo objetivo es establecer las cualidades polifacéticas de objetos, procesos, servicios y sistemas, en ciclos vitales enteros, por lo que es también el agente central de la humanización de tecnologías innovadoras y el factor crucial del intercambio económico y cultural.

Queda debidamente reconocido que el diseño industrial es, ante todo, una profesión intelectual y que, como tal, precisa obligadamente de una mínima base teórica, implícitamente enmarcada en su propia definición.

\section{Estrategias metodológicas elegidas}

Las estrategias metodológicas se basan en diferentes fases que aseguran un avance gradual. El trabajo de gabinete supone al principio una fase exploratoria, de consulta y recopilación documental, con revisión de la literatura sobre el tema propuesto desde las disciplinas de la historia del diseño industrial y la teoría del diseño, con el objetivo de profundizar en las bases conceptuales desde las que se parte, a través de los autores, teorías y proposiciones generales dentro de las cuales se sitúa este trabajo de indagación, en la selección de autores y productos a analizar y en la recopilación del material documental correspondiente.

Asimismo, se busca vincular el trabajo de gabinete con los antecedentes históricos que proporcionaron insumos para la realización del proceso de diseño y del producto concreto; y realizar un análisis valorativo de los casos de estudio, sus teorías sustentantes y su influjo en la conformación de nuevas líneas de pensamiento proyectual.

En segunda instancia, el trabajo de campo abarca la consulta de informantes clave: docentes y alumnos de talleres de diseño de los niveles 2,3 y 4 , seleccionados por ser los que han recibido los aportes de las asignaturas de Historia del Diseño Industrial y el Arte e Historia de la Industria Argentina, Sociedad y Cultura en forma completa. 
Mediante la observación, análisis y valoración de trabajos prácticos de los mencionados talleres de diseño, en forma conjunta entre los docentes de historia y teoría y los de los talleres, se procede a verificar la existencia de los conocimientos de historia y teoría del diseño como insumos proyectuales.

En una tercera etapa, el trabajo de gabinete se instala para el procesamiento de datos, es decir, su clasificación mediante codificación y tabulación, su análisis, interpretación y validación mediante instrumentos cuantitativos, más el informe de resultados y las propuestas didácticas de abordaje del proceso de diseño utilizando como insumos los conocimientos aportados por historia y teoría del diseño industrial.

Finalmente, un nuevo trabajo de campo posibilita la implementación de las estrategias diseñadas, la observación de los resultados obtenidos y la retroalimentación y ajuste en función de dichos resultados.

Las estrategias consistieron en una serie de trabajos hacia el interior de la cátedra de Historia del Diseño Industrial: uno práctico, con operaciones de diseño menores, utilizando la historia como insumo; otro de reflexión, desde un caso ejemplar afín valorado críticamente y en relación con el tema de proyecto en el Taller de Diseño; y uno de integración entre cátedras como estrategia principal. Este último incluyó, además, una actividad de reflexión como inmersión inicial en las experiencias lúdicas personales de la infancia, orientando el objetivo del ejercicio hacia el diseño de juguetes.

\section{Resultados}

El trabajo exploratorio de gabinete aportó valiosas y diversas miradas referidas al problema planteado que enriquecieron el enfoque primariamente trazado. Asimismo, durante el proceso de indagación se detectó la necesidad de ahondar en la cuestión de la teoría del diseño, ya que el enfoque de los diversos autores revisados variaba significativamente.

En este sentido, el aporte de Bonsiepe (1999) en su libro Del objeto a la interfase constituye el marco más apropiado. El autor deja aquí clara la idea de la necesidad del conocimiento teórico en la formación del diseñador, abordando el tema de la teoría desde las siguientes perspectivas: "La teoría del diseño y la práctica del diseño", "La caracterización de la teoría", "La legitimación de la teoría", "La visualización" y "La investigación sobre la relevancia del diseño".

De acuerdo con los resultados de las encuestas, se pueden inferir algunas conclusiones que aportan líneas de acción para optimizar la vinculación entre la historia del diseño industrial y los talleres de diseño industrial. 
Los estudiantes declaran tener un conocimiento alto tanto de historia como de teoría del diseño industrial, no obstante lo cual se registra un escaso porcentaje de alumnos que hayan aprobado los exámenes finales, de lo cual se deduce que el proceso de reflexión y maduración que requieren estas asignaturas no se ha completado debidamente.

Los fundamentos negativos a la pregunta cerrada 1, que solo se manifiestan en un $10 \%$ del total del alumnado encuestado, pero que no por ello deben dejarse de tener en cuenta, implican que se debe realizar un ajuste en contenidos y secuenciación del desarrollo de las historias en coordinación con los contenidos de los talleres, en tanto y en cuanto se consiga trabajar en forma integrada entre las dos áreas.

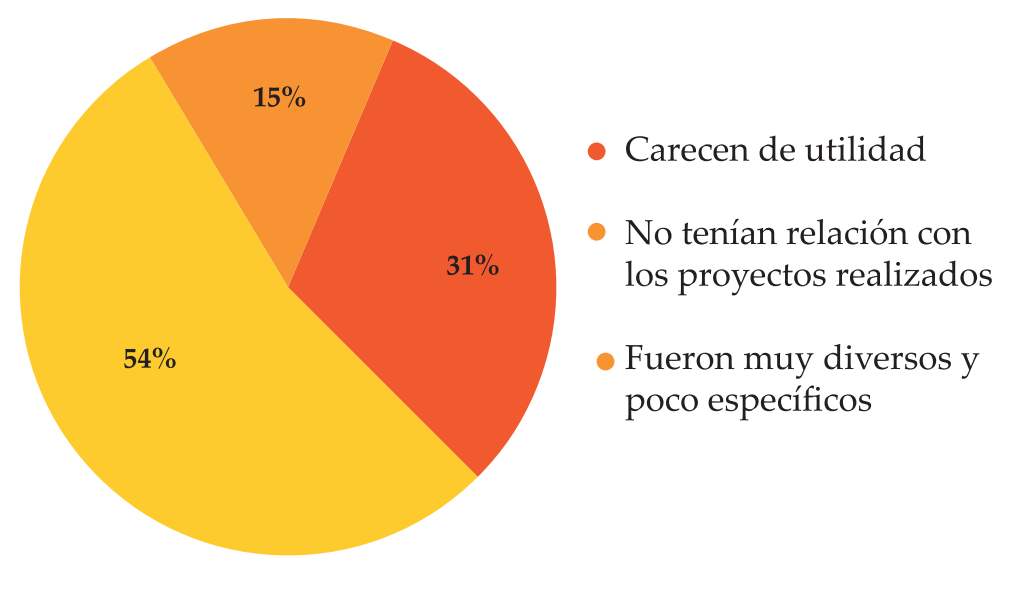

Figura 1. Fundamentos por NO a preguntas cerradas. 
Los fundamentos positivos a la pregunta cerrada 2 , que expresan el pensamiento de $90 \%$ de los alumnos participantes de la encuesta, revelan una extensa y profunda variedad de intereses que refuerzan la hipótesis de este trabajo de investigación: que una estrategia que permite a los alumnos tener una visión abarcadora de la cuestión es la práctica de trabajos integrados entre ambas áreas, tal como se implementó en los meses de setiembre y octubre de 2018.

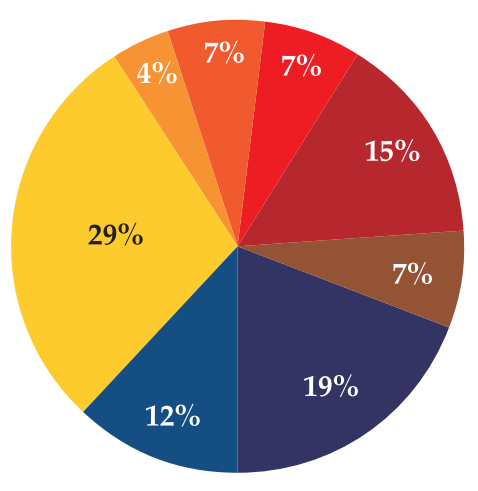

- Conocer relación entre arte, diseño, sociedad e industria

- Conocer la teoría de los diseñadores en sus objetos

- Conocer la evolución de los objetos y sus tecnologías

- Incorporar datos para fundamentar proyectar y diseñar

- Constituye una guía en el proceso creativo

- Procurar antecedentes

- Aprovechar los aportes del análisis de producto

- La estética de diferentes movimientos

Figura 2. Fundamentos por SI a preguntas cerradas.

Fuente: autores.

Los resultados a las preguntas 3,4 y 5 son similares, por lo que se interpreta que habría que poner atención a las respuestas "Podrían mejorar" y "NS/NC" con el objetivo de optimizar las prácticas pedagógicas. 


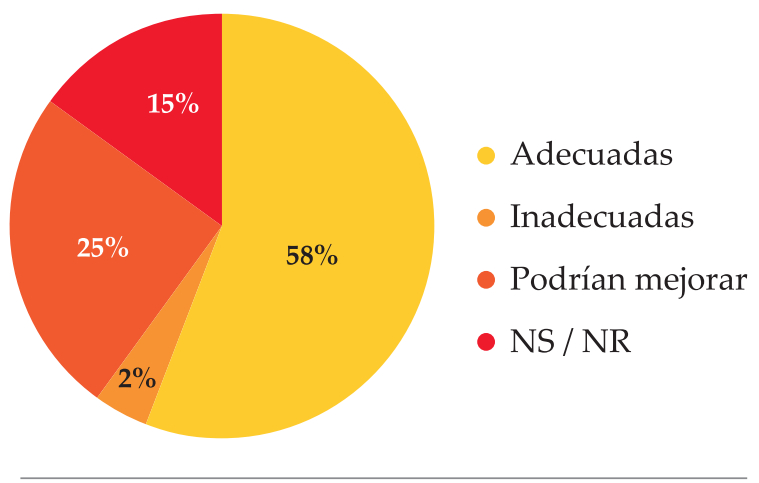

Figura 3. Estrategias de enseñanza.

Fuente: autores.

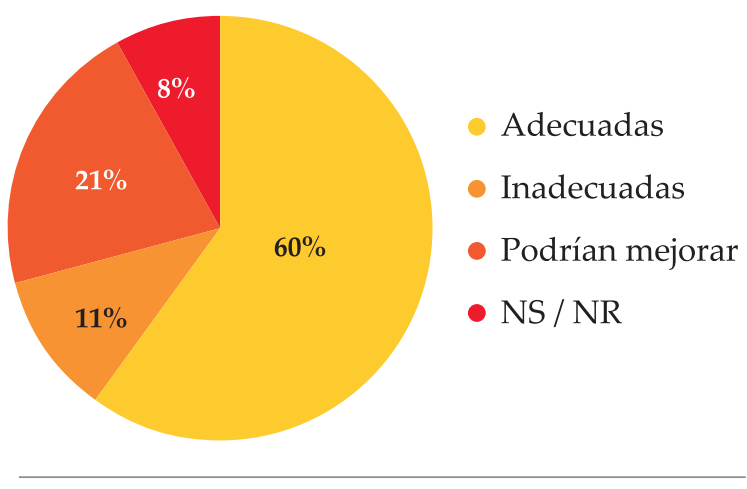

Figura 4. Estilo de comunicación.

Fuente: autores.

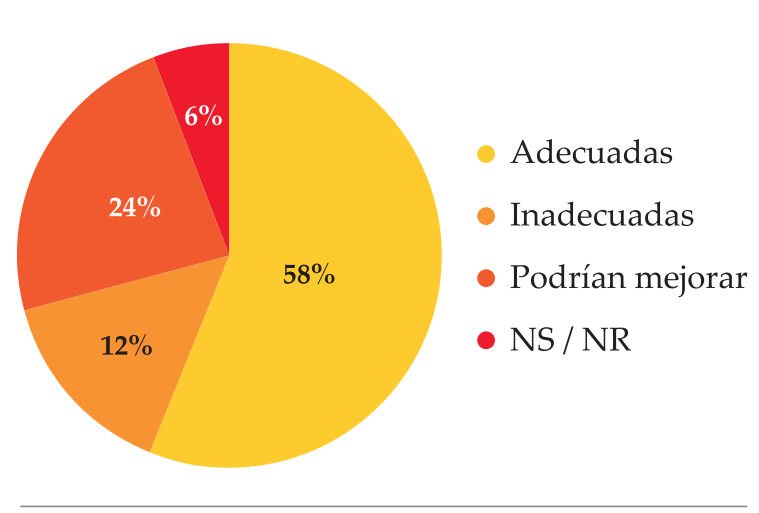

Figura 5. Criterios de evaluación. Fuente: autores. 
Los resultados de las respuestas a las preguntas 6 y 7 muestran con claridad la necesidad de trabajar integradamente entre los docentes de los talleres de diseño industrial con los de Historia del Diseño Industrial.

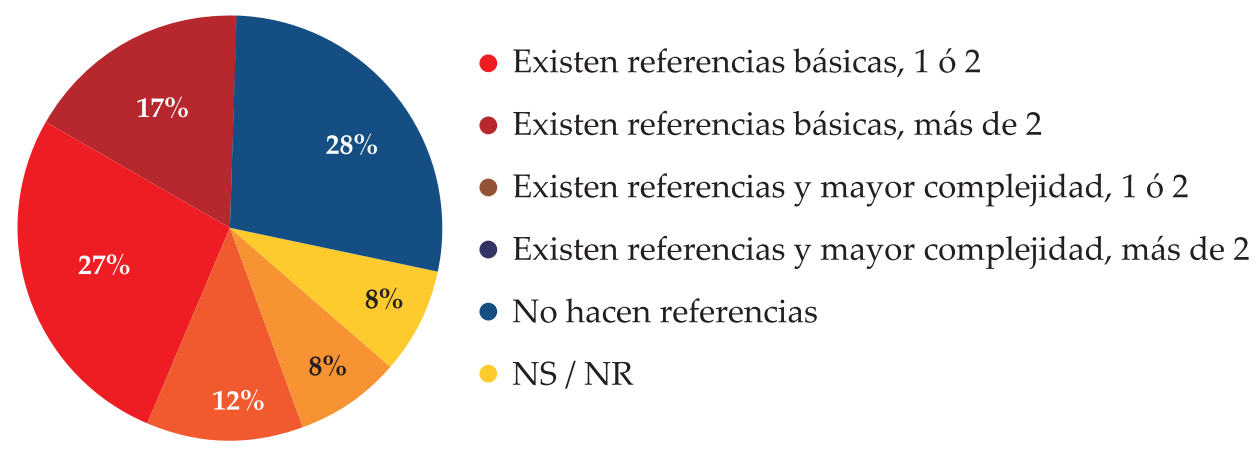

Figura 6. Referencias en talleres sobre Historia DI.

Fuente: autores.

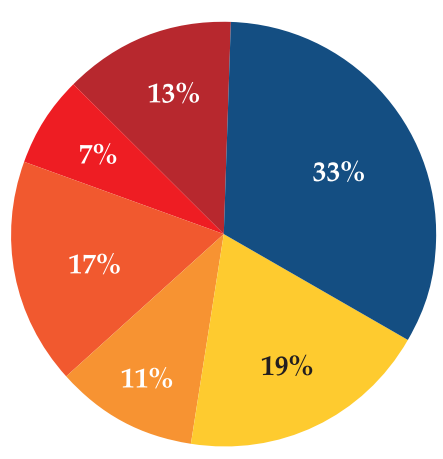

- Sin problemas

- No se relaciona con el trabajo en taller y no se utilizan

- Eficiente si se dispone de antecedentes suficientes

- Requiere comprensión y asociación al proyecto $\mathrm{p}$ / ser útil

- Amplia variedad de diseños y autores

- NS / NR

Figura 7. Aplicación en taller de conocimiento de Historia DI. 
Finalmente, la información proporcionada por las respuestas a la pregunta 8 no hace más que mostrar con claridad la preeminencia de internet como fuente de información.

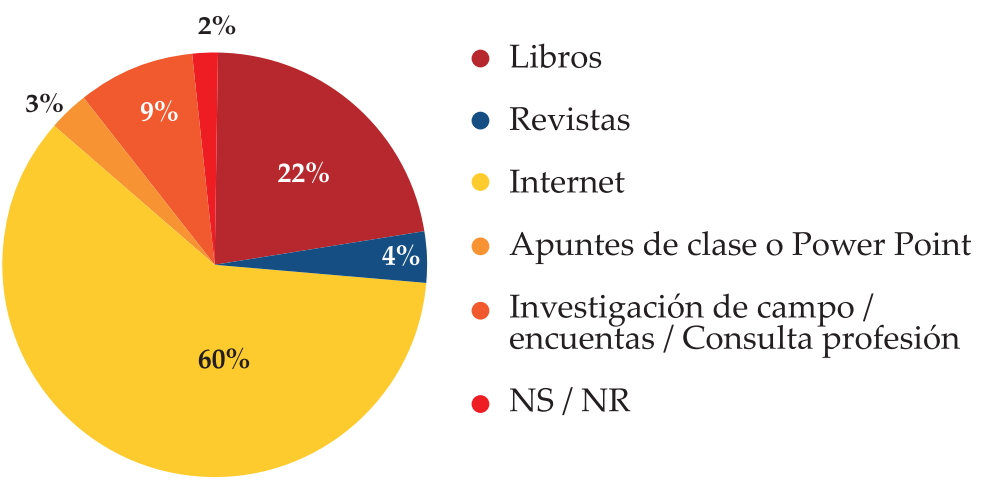

Figura 8. Fuentes utilizadas en la investigación.

Fuente: autores.

Respecto de los aportes de los docentes de taller, sus respuestas son coincidentes en que los enfoques que se plantean en los talleres de diseño industrial son eminentemente prácticos, aunque uno de los docentes sostiene que propone como parte del abordaje del proyecto la comprensión del contexto cultural.

Asimismo, y con algunas variaciones de grado entre las respuestas, los docentes observan que hay muy pocos aportes del resto de las asignaturas de la carrera. Del mismo modo, y referido a la investigación tanto en historia como en teoría, solo la vinculan con la búsqueda de antecedentes.

Todo esto expresa la necesidad de un trabajo transversal entre los equipos docentes de la carrera, con la colaboración del equipo de orientación pedagógica de la universidad, para acordar estrategias de vinculación y acuerdos a fin de superar la concepción/realidad de compartimentos estancos para cada asignatura y/o área de conocimiento. 
Con este propósito, se plantearon tres trabajos de integración entre los talleres de diseño industrial y la historia del diseño industrial. El primero se desarrolló entre las materias Taller II e Historia del Diseño, referido a la cultura del sentarse, y a tal efecto se propuso reflexionar sobre un texto de Peter Opsvik (2009), poniendo en evidencia la evolución de la cultura del sentarse para descubrir así las modificaciones derivadas de la incorporación de los avances de la medicina y la ergonometría en beneficio de un más acabado diseño de los asientos.

Esta actividad se diseñó de manera informal, por iniciativa del docente de Historia del Diseño Industrial, como entrada a la integración de la mirada histórica con el quehacer específico del diseñador industrial. Se pone así, en consideración el proceso propuesto por Opsvik, en su perspectiva histórica y desde las experiencias sobresalientes que desembocaron en diseños particulares en la cultura del sentarse durante los siglos XIX y XX, y se aborda críticamente este sesgo hasta llegar a la actualidad.

De esta manera se pretende construir una matriz reflexiva-análoga con el alumno, de manera que logre avizorar la importancia de la dimensión histórica del diseño de productos en casos emblemáticos. Este mismo diseñador nórdico representa un caso ejemplar, que se nutre del insumo del pasado para construir un problema de diseño en el presente.

El segundo trabajo se desarrolló durante la cátedra de Historia del Diseño Industrial de segundo año, con la intención de integrar el saber histórico como bagaje conceptual para enriquecer las miradas y/o abordajes contemporáneos sobre problemas generales de diseño industrial. Se utilizaron en este caso insumos provenientes del movimiento 
Pop de la década del sesenta del siglo XX, con dos alternativas de resolución: o bien un escenario posible de diseño de productos contemporáneos sobre el que se puedan transferir aquellos principios del Pop, o se toman productos presentes para evaluar el carácter residual de los principios antes aludidos.

Esta experiencia ha dado muy buenos resultados, a partir de los procesos de reflexión y crítica realizados por los alumnos en equipo, con algunas propuestas valiosas desde lo conceptual

El tercer trabajo se planteó con la intención específica de consolidar un marco institucional de integración entre Historia del Diseño Industrial y el Arte y Taller de Diseño I, y consistió en un ejercicio particular cuyas instancias procesuales fueron diseñadas conjuntamente entre cátedras, para el curso del primer nivel, tanto de Santa Fe como de Rosario. Esto permitió anclar la investigación en una muestra de 83 alumnos pertenecientes a las sedes de Santa Fe y de Rosario de la Universidad Católica de Santa Fe, de los niveles 2.do, 3.ro y 4.to.

Además de la propuesta de una guía metodológica, elaborada en conjunto por docentes de talleres e historia, se previeron estrategias hacia el interior de las historias como mecanismo permanente de integración, y como metodología natural para el abordaje de las cuestiones históricas en perspectiva proyectual.

El proyecto de este trabajo práctico consistía en el diseño y desarrollo de un juguete educativo para niños o niñas, dentro de un rango de edad a definir entre $\operatorname{los} 0$ y los 12 años.

El trabajo era diseñar y desarrollar un producto para cualquiera de las siguientes categorías: ciencia, libro y escritura, arte y creación, construcción, actividades.

Se trabajó en equipos de estudiantes, los cuales, a su vez, fueron conformados por miembros del equipo docente. Por tratarse de un trabajo de integración, el mismo fue desarrollado de manera conjunta con la asignatura Historia del Diseño Industrial y del Arte.

La participación de los docentes de esa cátedra se dio en diferentes etapas del proceso de diseño, por lo menos en cuatro instancias en el ámbito propio del Taller de Diseño, aunque también en el marco del dictado de la materia de Historia, con aportes sostenidos desde los contenidos específicos, las tutorías, etc. 
1- Lanzamiento del trabajo práctico: Aquí se propone además un ejercicio de introspección hacia el mundo de lo lúdico, proyectando una mirada introspectiva y retrospectiva respecto de las funciones del juego y los juguetes. Estas reflexiones suponen la inmersión en el universo de lo lúdico para el niño, desde las experiencias personales como primera aproximación hacia la construcción del problema de diseño. Los alumnos grafican, explicitando en los juguetes de su infancia algo de ese universo lúdico, apelando a la memoria emotiva como clave esencial para dar inicio a la actividad.

\begin{tabular}{|l|}
\multicolumn{1}{|c|}{ Reflexiones iniciales disparadoras: } \\
\hline a. Caracterice su universo lúdico de niño/a. \\
\hline b. Traiga a la memoria algún juguete de su infancia. \\
\hline c. Destaque sus cualidades, cualesquiera que estas sean. \\
\hline $\begin{array}{l}\text { d. Identifique las contribuciones que estos juguetes han tenido en su } \\
\text { aprendizaje o formación. }\end{array}$ \\
\hline $\begin{array}{l}\text { e. ¿Qué importancia les asignaría a los juguetes en la etapa de niñez? } \\
\text { Fundamente. }\end{array}$ \\
\hline ¿Qué piensa de los juguetes contemporáneos? \\
\hline
\end{tabular}

Tabla 3. Funciones del juego y el juguete. Una mirada introspectiva / retrospectiva.

Fuente: autores.

2- Entrega de programa de proyecto e investigación: Se validan aquí, desde una dimensión histórica, la conveniencia de los antecedentes seleccionados, el abordaje, la profundidad y la complejidad en la mirada histórica, y la posibilidad de relacionar problemas y soluciones del pasado con inquietudes, demandas y necesidades presentes. Se ponderan igualmente los insumos bibliográficos aportados por las cátedras de Historia del Diseño Industrial y Taller de Diseño, y la utilización de los mismos en la construcción de un marco conceptual que fundamente las instancias ulteriores. 
3- Presentación de diseños conceptuales: Se valoran en estos el impacto del abordaje de la dimensión histórica del problema, y cuánto ha contribuido la etapa anterior a la construcción del problema de diseño en sentido de la complejidad. Se plantean cuestiones relacionadas con el marco conceptual desarrollado y su impacto en los diseños de esta fase.
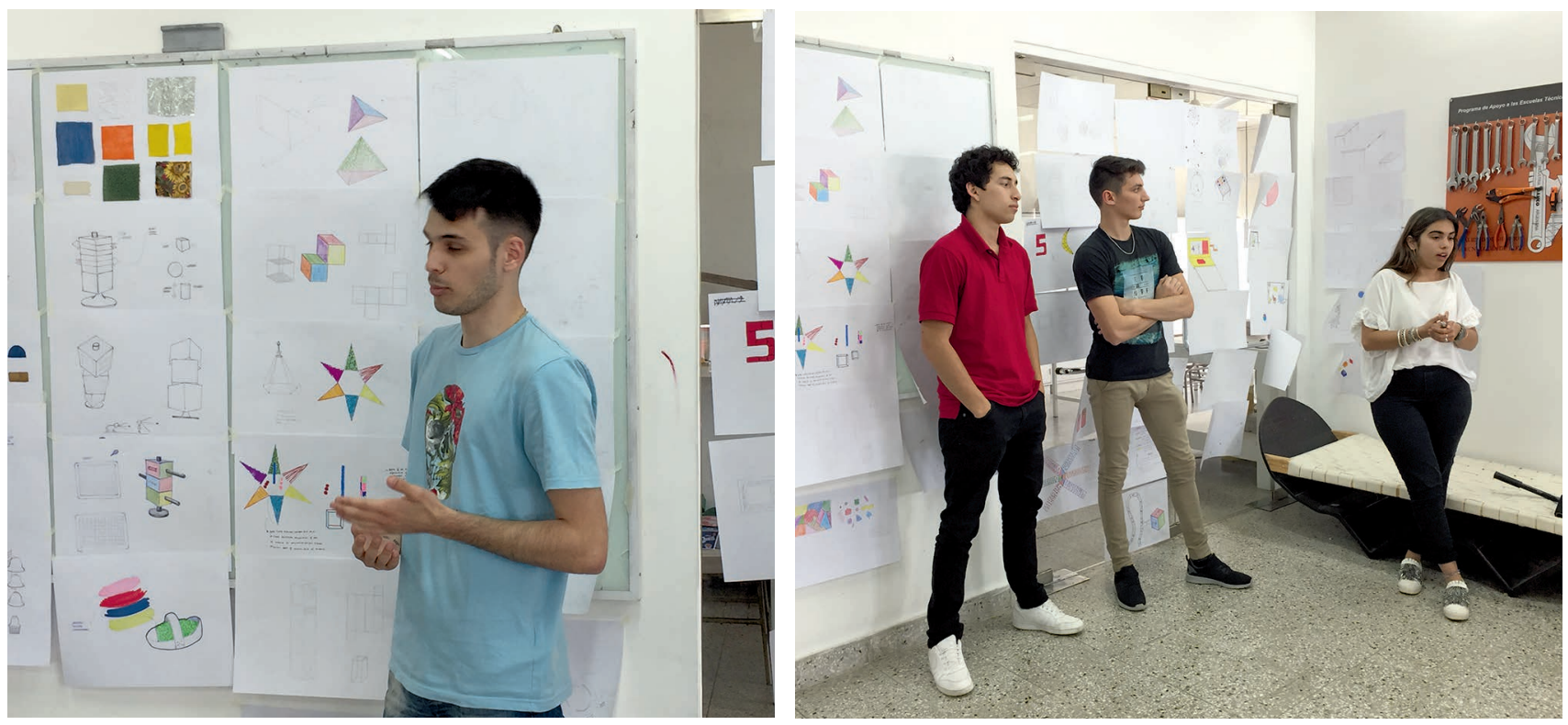

Figura 9. Presentación de diseños conceptuales: la enchinchada expone aspectos del proceso de diseño por equipos.

Figura 10. Presentación de diseños conceptuales: el equipo expone los criterios de diseño, las iteraciones y sus fundamentos. 
4- Presentación de proyecto completo final: En los prototipos finales, el influjo de la historia del diseño industrial se presenta a modo de antecedentes del tema de proyecto y a partir de algunas consideraciones que podrían interpretarse como derivadas de un proceso de construcción gradual y creciente del problema de diseño desde la historia, lo cual desemboca en un abordaje singular.
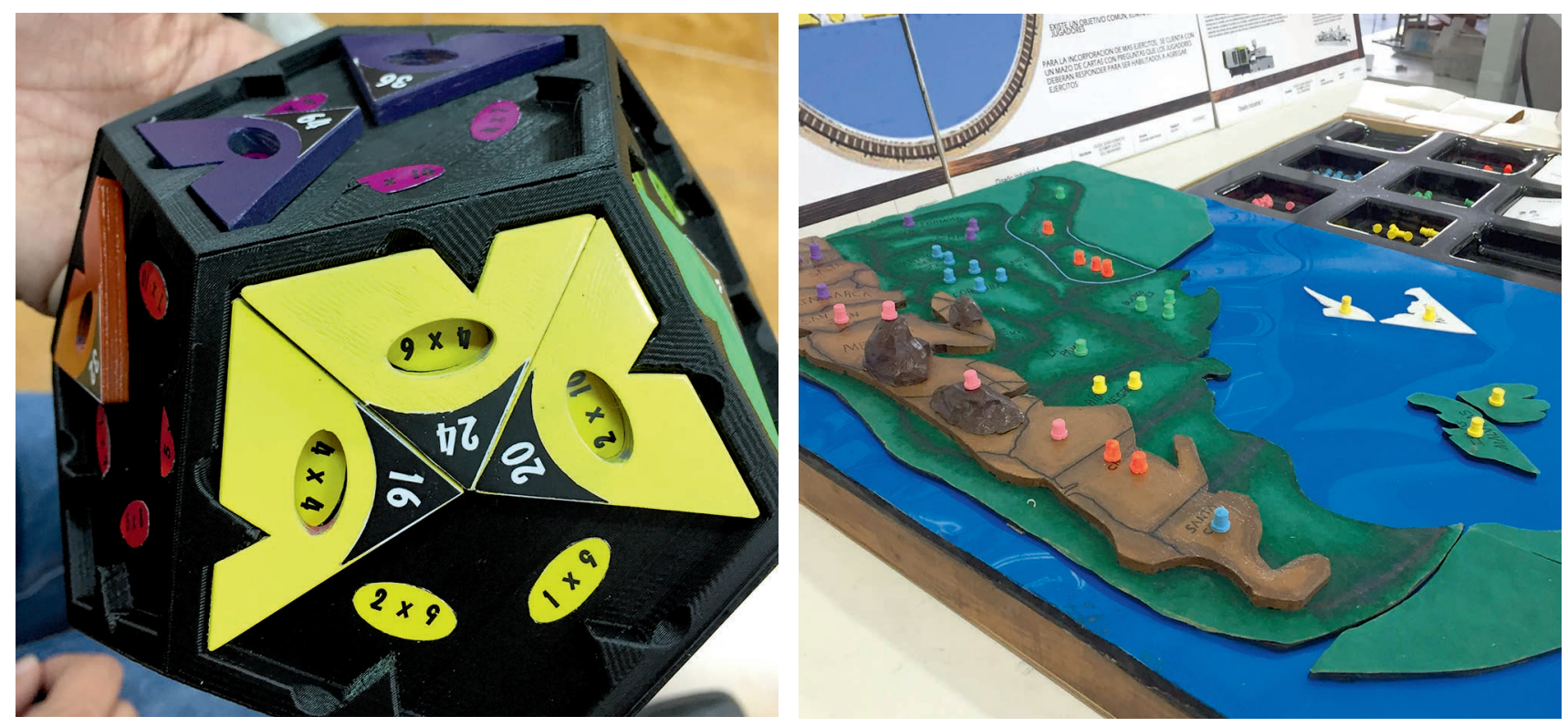

Figura 11. Proyecto 1: Mathedron. Juguete para niños de 7 a 9 años para aprender las tablas de multiplicación.

Figura 12. Proyecto 2: T.E.G. Alternativa del juego de estrategia para el aprendizaje de la geografía. 

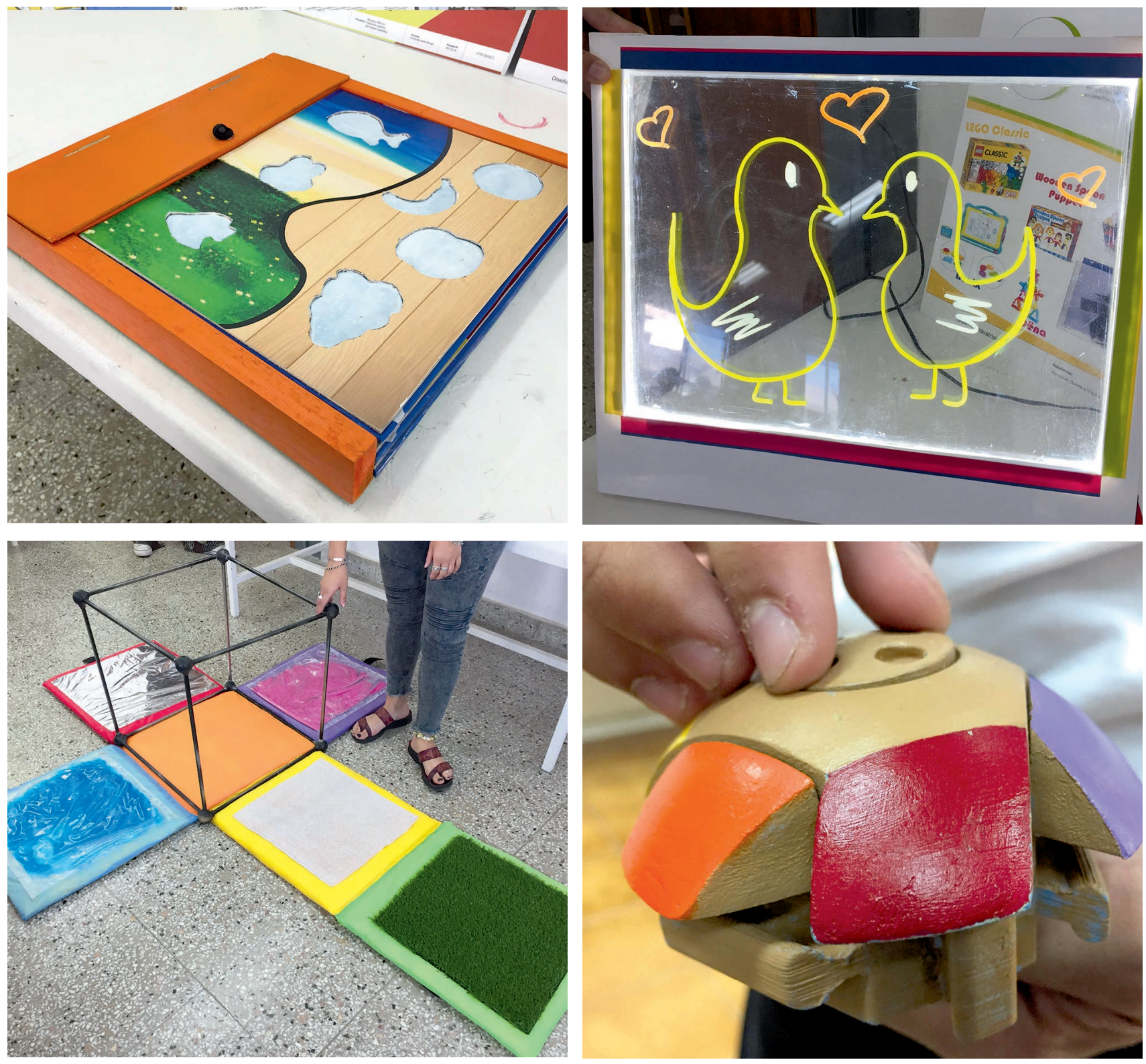

Figura 13. Proyecto 3: Figure Board. Juguete para niños de 2 a 4 años para aprender a relacionar figuras. Figura 14. Proyecto 4: Pantalla de luz. Juguete para niños de 4 a 6 años para explorar el dibujo y formas con luz. Figura 15. Proyecto 5: Juego de aprendizaje. Espacio de juego con alfombras texturadas para niños de 6 a 12 meses.

Figura 16. Proyecto 6: Chromatic Sphere. Juego de habilidad y encastre por colores para niños de 8 a 12 años. 
Presentación de prototipos finales: los equipos exponen las características del producto, las tecnologías utilizadas y los fundamentos desde la Historia del Diseño Industrial como insumo.
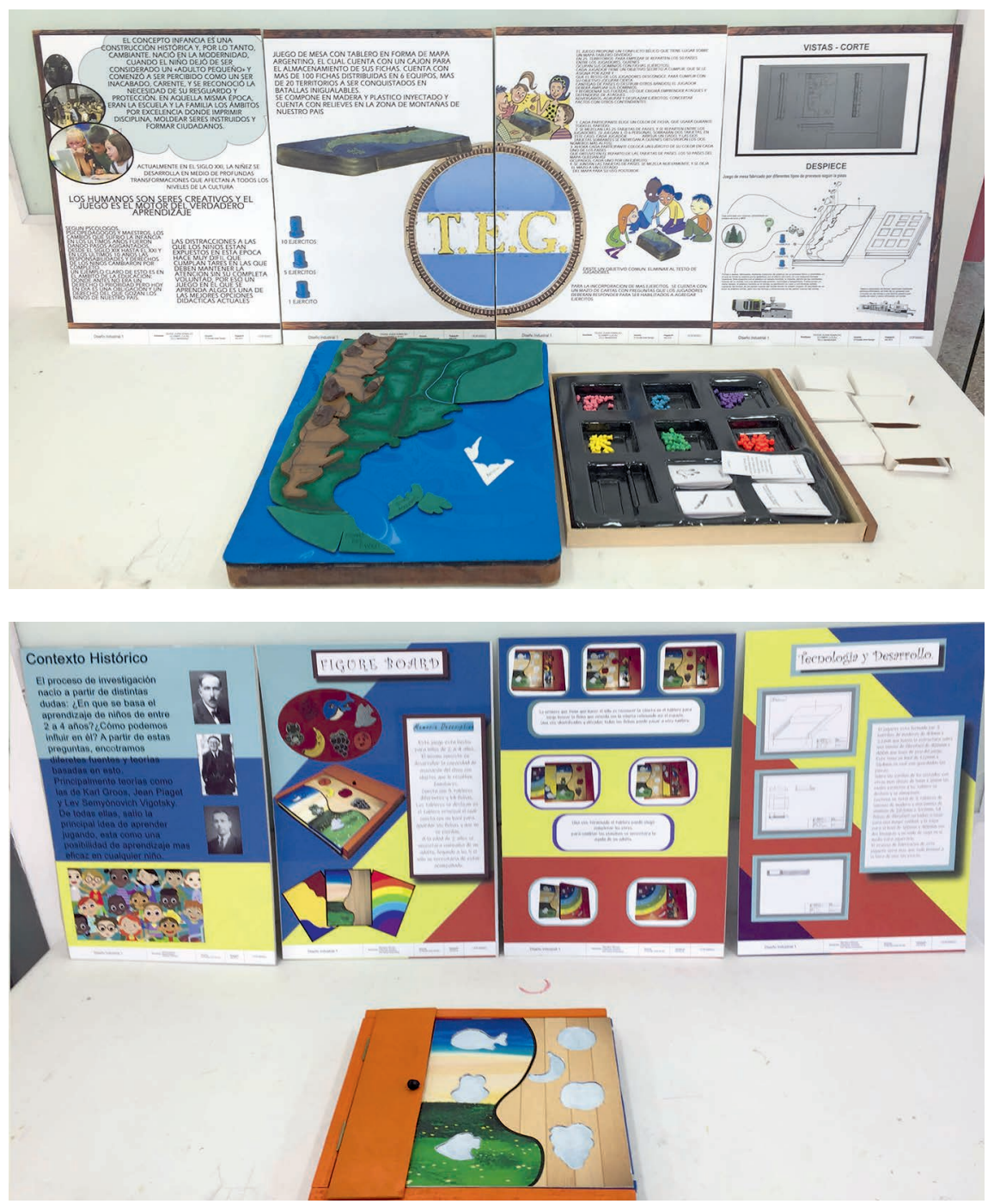

Figura 17. Lámina correspondiente al proyecto 2: T.E.G. Con los lineamientos de proyecto se solicitó la realización de 4 láminas representadas en paneles formado A2 para presentación, conteniendo información relativa al proyecto.

Figura 18. Proyecto 3: Figure Board. Las 4 láminas de cada proyecto contienen información sobre: $1^{0} \mathrm{el}$ contexto histórico como fundamento, $2^{\circ}$ memoria descriptiva e imágenes, $3^{\circ}$ secuencia de uso, $4^{\circ}$ tecnología. 
Cabe aclarar que se hicieron seguimientos por equipos antes de las entregas previstas en el cronograma, revisando oportunamente los casos elegidos, los enfoques conceptuales y la bibliografía seleccionada. Este feedback resulta de vital importancia, ya que es en la reflexión conjunta que se puede arribar a instancias superadoras.

Se recuperan los textos de historia del diseño industrial como recursos para poder situar espacio temporalmente y comprender los enmarques culturales, respecto de los casos de estudio. De la propuesta bibliográfica temática se seleccionan textos para su consideración como lecturas específicas.

Desde la historia del diseño se elabora una presentación integradora, seleccionando para el análisis casos, diseñadores y productos contenidos en el programa de la materia, que resulten ejemplares para su consideración en este ejercicio.

Si bien existe registro suficiente de una mayor producción de juguetes en casi todas las etapas históricas atravesadas por los contenidos del programa de Historia del Diseño Industrial y el Arte, se muestra una casuística del siglo XIX, con juguetes típicos de la época. Mediante la pregunta: ¿qué aspectos del niño habrían desarrollado estos juguetes?, se pretende una reflexión desde el pasado y según las características de los juguetes de aquellos tiempos, con el objetivo de empezar a construir el problema de diseño.

Se solicita a los alumnos, así mismo, que recuperen del dictado de la materia Historia del Diseño Industrial y el Arte contenidos de enmarque cultural para los diferentes temas y problemas inherentes al diseño de productos, los diferentes movimientos o corrientes, los diseñadores y los puntos de inflexión hacia nuevos paradigmas durante el cambio del siglo XIX al siglo XX para, de esta manera, situar los casos específicos en su correcta ubicación espacio temporal. 
A continuación, se exponen los objetivos presentados oportunamente en el programa de Historia del Diseño Industrial y el Arte, para situar este ejercicio de integración:

- Introducir a los estudiantes en la categorización de los conceptos históricos y de diseño.

- Comprender las causas que motivaron las diferentes manifestaciones artísticas, creativas, estéticas y sus consecuencias.

- Promover en los estudiantes la integración de la mirada histórica con el quehacer específico del diseño industrial.

- Desarrollar dispositivos para que el estudiante logre visualizar las características cambiantes de la cultura del producto en la movilidad del inicio del mundo contemporáneo.

En este sentido, la historia y los casos emblemáticos conforman una vía de aprendizaje, que mediante la reflexión y la crítica se proponen como instancias que pueden contribuir a la construcción de la oportunidad de diseño; sin embargo, es claro que la historia tiene otros tiempos para poder anidar en el alumno y convertirse en insumo operativo para el proceso de diseño.

\section{Evaluación de la actividad de integración}

Se ha realizado, a su vez, la evaluación del ejercicio integrador de manera procesual, si bien se considera especialmente la entrega final.

En las entregas se percibe que en la mayoría de los casos se ha hecho algún esfuerzo en la indagación respecto de los antecedentes, tratando de integrar la mirada histórica al proceso de diseño. Se han considerado casos diversos como estudio de antecedentes, investigando en otros campos como el de la pedagogía o la psicología del niño, pero teniendo en cuenta de manera muy limitada las cuestiones culturales de época, para poder comprender las relaciones de causa y efecto en los casos analizados.

Si bien se han utilizado recursos de internet, en general se nota que se ha copiado directamente sin citar las fuentes. Se transcribe casi absolutamente todo como si fuera producción propia. Quizás por ser el primer nivel de la carrera, y en ausencia de formación específica en lo que a metodología de la investigación se refiere, es que los trabajos adolecen de rigor metodológico. La bibliografía, cuando aparece, no está adecuadamente citada, ya sea de libros o artículos de internet. Algunas presentaciones incluso no están identificadas por sus autores, lo que es evidencia de cierta despreocupación de los aspectos formales de la investigación. 
Existe una aproximación a la problemática desde recursos de la web en la definición del juego y el juguete, los tipos de juegos y juguetes, y la función pedagógica de los mismos, entre otros, así como una mención a los casos de estudio que se han visto en la propuesta integradora, casi de manera forzada, si bien escasamente se tiene una construcción del problema utilizando el insumo histórico como matriz reflexiva. Se ha pedido a los estudiantes en las correcciones que buscaran en la historia insumos vinculados a la problemática elegida, para así establecer algún tipo de relación más directa con los requerimientos del ejercicio.

En general se reutilizaron los insumos de la presentación realizada por la cátedra de Historia del Diseño Industrial y el Arte, haciendo referencia solo a los casos desarrollados en clase, pero sin relacionarlos reflexivamente con los problemas estudiados según las prerrogativas del ejercicio de diseño.

Dadas estas dificultades, se esperaba poder desarrollar estrategias hacia el interior de las historias que posibilitaran una mayor comprensión y un mejor aprovechamiento de los insumos históricos, orientándolos hacia una construcción eficiente del problema de diseño.

\section{Conclusiones y recomendaciones}

Desde las actividades de integración llevadas a cabo en distintos ámbitos y de acuerdo con los resultados obtenidos, se propone, en una instancia de integración futura, proveer por medio de las estrategias conjuntas una mejor revisión de las etapas realizadas o concluidas, en la mirada del alumno, para comprender que el proceso de diseño supone un camino no lineal, sino espiralado, según el cual siempre es posible volver sobre el camino trazado para revisar y/o readecuar dicho proceso, a partir de aproximaciones críticas, por lo que será siempre en un nivel diferente y cada vez más próximo al constructo final.

Se espera que estos aportes refuercen la actitud de los estudiantes, en función de lograr una mayor proactividad y diligencia a la hora de detectar los problemas reales existentes sobre los cuales trabajar en el proyecto.

Las actividades de integración deben realizarse de manera sistemática y de un modo periódico tal que el alumno internalice dicho camino como un elemento fundamental en el proceso de diseño de productos. Así mismo, se debieran suministrar mecanismos 
de integración entre las diferentes áreas de la formación curricular, no solo con las historias del diseño industrial, para así resolver el problema de la compartimentación del conocimiento en la formación disciplinar.

Con tales expectativas, el trabajo conjunto e integrado de los docentes de las diferentes áreas curriculares resulta un momento muy importante en la formulación de las estrategias de enseñanza y aprendizaje del diseño.

En el caso particular de la historia del diseño industrial, el abordaje gradual y creciente de primer a segundo año supone una continuidad del proceso de integración, actividad que debiera prolongarse incluso a todo lo largo de la formación académica.

La tarea fundamental desde la historia del diseño industrial supone una articulación permanente con problemas inherentes al proceso de diseño en taller, haciendo visibles las relaciones posibles entre pasado y presente, construyendo los problemas desde una dimensión teórica. Semejante proceso debe desarrollarse de manera diacrónica, por lo que el trabajo sostenido deviene esencial para el logro de los objetivos previstos.

De esta manera, se pretende consolidar, en los ciclos académicos por venir, una metodología mancomunada de trabajo docente para la planificación de las estrategias de integración y el fortalecimiento, tanto en los espacios de historia como en taller, de las instancias de discusión y debate sobre aquellos aspectos teóricos comprometidos con el problema de diseño. Para esto es necesario lograr acuerdos entre docentes respecto a los recursos bibliográficos más adecuados, sobre una base conceptual determinada.

Asimismo, es necesario trabajar con un sentido diacrónico y de gradualidad creciente en la profundidad y complejidad de los abordajes teóricos, y planificar conjuntamente las articulaciones posibles o deseables entre un área y la otra, dado el tema de proyecto/diseño.

Del mismo modo, se propone implementar en la cátedra de Historia del Diseño Industrial actividades de diseño conceptual breves, tomando el pasado como variable e incorporando la dimensión crítica como insumo fundamental para la resolución de problemas de diseño, para incorporar de este modo en la carrera de Licenciatura en Diseño Industrial una cátedra a desarrollarse durante el primer año de cursado sobre metodología de investigación. 


\section{REFERENCIAS BIBLIOGRÁFICAS}

Bonsiepe, Gui (1999). Del objeto a la interfase, mutaciones del diseño. Argentina: Ediciones Infinito.

Ferrer Forés, J. (2009). El proyecto arquitectónico como material de investigación. Departamento de Proyectos Arquitectónicos. Escuela Técnica Superior de Arquitectura de Barcelona. Universitat Politècnica de Catalunya.

García Trompa, J. A. (2006). Repensar el objeto. Nuevas dinámicas del Diseño Industrial —un manifiesto-, Diseño en Palermo. [Ponencia en Encuentro Latinoamericano de Diseño]. Recuperado de: https://fido. palermo.edu/servicios_dyc/publicacionesdc/vista/detalle_publicacion.php?id_libro=13

Guigosa Fragoso, V. (2006). La investigación en diseño. Actas de Diseño 1, 76-77. Recuperado de https://fido. palermo.edu/servicios_dyc/publicacionesdc/vista/detalle_publicacion.php?id_libro=13

Kruft, H-W. (1990). Historia de la teoría de la arquitectura, vol. 1. Madrid, Alianza.

Maldonado, T. (1997). Escritos preulmianos. Buenos Aires, Ediciones Infinito.

Ochoa, A., y Agusto, F. (2014). La historia del diseño industrial. Una herramienta de lectura crítica en la cultura proyectual [ponencia presentada al VI Encuentro de Docentes e Investigadores en Historia del Diseño, la Arquitectura y la Ciudad]. Recuperado de: http://sedici.unlp.edu.ar/handle/10915/43018/discover

Opsvik, P. (2009). Rethinking sitting. New York, W.W Norton \& Company.

Reinders, A. H.; Diehl, J. C.; Brezet, H. (2013). The Power of Design: Product Innovation in Sustainable Energy Technologies. John Wiley \& Sons, Ltd. United Kingdom. Recuperado de: https://books.google.com.ar/ books?id=twzqIr9opHgC\&pg=PA11\&dq=icsid+industrial+design+humanization\&hl=es-419\&sa=X\&v ed=0ahUKEwjCtKXL6_3jAhWWHLkGHYZTA98Q6AEIRzAE\#v=onepage\&q=icsid\%20industrial\%20 design $\% 20$ humanization\& $\mathrm{f}=$ false

UNESCO (1980). El niño y el juego. Planteamientos teóricos y aplicaciones pedagógicas. Recuperado de: https://unesdoc.unesco.org/ark:/48223/pf0000134047

Zamora Sarmiento, L. M. (2015). ¿Diseñador o investigador? Nuevos roles del profesional en diseño. Actas de Diseño 19, 143-146. Recuperado de https://fido.palermo.edu/servicios_dyc/publicacionesdc/vista/ detalle_publicacion.php?id_libro=541 